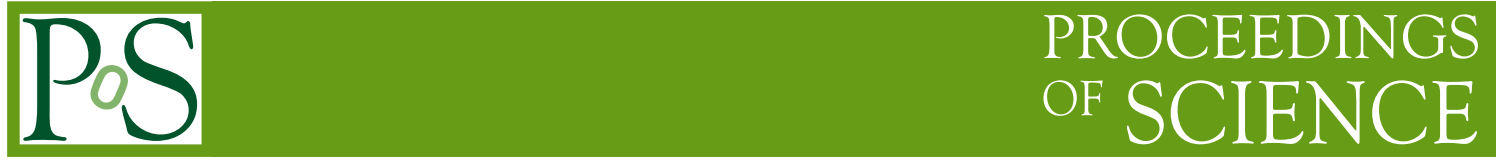

\title{
New physics in top sector at the Tevatron
}

\author{
Ford Garberson*广 \\ Yale University \\ E-mail: fgarb@fnal.gov
}

XVIII International Workshop on Deep-Inelastic Scattering and Related Subjects April 19 -23, 2010

Convitto della Calza, Firenze, Italy

${ }^{*}$ Speaker.

${ }^{\dagger}$ On Behalf of the CDF Collaboration 


\section{Introduction}

By now, many thousands of top quarks have been produced at the Fermilab Tevatron Collider. With such high statistics, many precision studies have become possible. In particular, many searches for new physics in the top sector have been performed recently at the CDF and D0 Collaborations. This note will detail some of them, while others will be discussed in a related proceedings note [1]. Most of the analyses we discuss will be directed searches in which a new physics model or class of models is hypothesized, and where the data is analyzed for consistency with the model. We will also discuss one analysis which is simply a study of the top charge directional asymmetry, but which shows an intriguing possible disagreement with Standard Model expectations. Many of these analyses start by reconstructing the top quarks from their decay products, usually after selecting them in the single lepton or the dilepton decay channels. For details on these reconstruction algorithms, please see the proceedings notes on Tevatron top mass measurements [2].

Many of the analyses discussed in these proceedings are in progress and are in the process of adding further data. The reader is advised to consult the public top group webpages for the experiments for the latest results [3].

\section{Searches for Resonant $t \bar{t}$ Production}

Many new physics models predict the existence of heavy particles that would decay into topquark pairs. In contrast to Standard Model $t \bar{t}$ production, these resonances would cause a peak in the invariant mass of the reconstructed $t \bar{t}$ system. Regardless of the specifics of the theory, this peak should be visible on top of the exponentially decaying Standard Model background.

Both CDF and D0 have performed recent searches where they reconstruct the invariant mass of the $t \bar{t}$ system to search for evidence of new physics. D0 has performed a search in the single lepton channel with $3.6 \mathrm{fb}^{-1}$ of integrated luminosity [4], and CDF has performed a similar search in the all hadronic channel $\left(2.8 \mathrm{fb}^{-1}[5]\right)$. For D0 the missing neutrino momentum is determined according to the measured missing transverse energy and a W-mass constraint. While analyses in the single lepton channel conventionally require four reconstructed jets, D0 allows events to be accepted in which only three jets are reconstructed. This allows for significantly higher signal acceptance, especially for high mass resonances where the tops are expected to be highly boosted and have merged jets in their decay products. For CDF the primary challenge is to reduce the enormous QCD background. It does this by applying a neural network event selection. It further attempts to correct the reconstructed jet momenta by incorporating the reconstructed jet momentum resolution functions into their calculations of the most likely reconstructed $t \bar{t}$ mass.

In the end, both CDF and D0 observe invariant mass distributions that are consistent with the Standard Model. To test the sensitivity of their methods, they pick a common example scenario of a leptophobic topcolor resonance with width $\Gamma_{Z^{\prime}}=0.012 M_{Z^{\prime}}$. With their respective analyses, CDF excludes this model up to a $Z^{\prime}$ mass of $805 \mathrm{GeV} / \mathrm{c}^{2}$ and D0 excludes the model up to a $Z^{\prime}$ mass of $820 \mathrm{GeV} / \mathrm{c}^{2}$.

\section{Searches for Supersymmetric $\tilde{\tilde{t}} \overline{\tilde{t}}$ Production}

Some supersymmetric models predict that, due to the heaviness of the top quark, the lightest 
supersymmetric stop quark will be lighter than any other squark, and may even be lighter than the top quark itself. This opens up a number of possible decay modes that could exist and be discovered in the top sector. Both CDF and D0 have recently performed searches for $\tilde{t} \rightarrow b X$.

D0 directs its search ([6], $3.1 \mathrm{fb}^{-1}$ ) towards the scenario where the chargino is too heavy to exist in the decay chain, in which case the stop is likely to decay directly $\tilde{t} \rightarrow b \tilde{v}^{0} \bar{l}^{+}$. In this situation, $\tilde{t} \tilde{t}$ production will, of course, look very similar to dileptonic $t \bar{t}$ production. It can only be separated from standard model $t \bar{t}$ events by analyzing the kinematic distributions of the decay products. D0 chooses to search for this signature in a channel where they require exactly one electron and one muon to kill most of the $\mathrm{Z}$ background, and enforce strict cuts on the magnitude and direction of the missing transverse energy to further reduce the remaining $Z \rightarrow \tau \bar{\tau}$ and QCD backgrounds. Jet selection cuts are deliberately left very loose, and $b$-tagging is not applied to retain statistics. They then search for evidence of supersymmetry simultaneously in two discriminating variables: $H_{T}$ (the scalar sum of the transverse momenta of all jets in the event), and $S_{T}$ (the same, but additionally including the leptons and the missing transverse energy). Failing to see any evidence of signal, they set limits on the model as a function of hypothesis stop and sneutrino masses. Assuming a $100 \%$ branching fraction of top squarks to the decay chain $\tilde{t} \rightarrow b \tilde{v}^{0} \bar{l}^{+}$, top squark production is now excluded over almost the entire kinematically-allowed phase space.

In contrast, CDF directs its search ([7], $2.7 \mathrm{fb}^{-1}$ ) toward the case where the chargino is light, and the decay $\tilde{t} \bar{t} \rightarrow b \tilde{\chi}^{+} \bar{b} \overline{\tilde{\chi}}^{-} \rightarrow b \bar{b} l^{-} \bar{l}^{+} v \bar{v} \tilde{\chi}^{0} \tilde{\chi}^{0}$ is dominant. Again, this process is similar to Standard Model dilepton $t \bar{t}$ decays, except that this time the missing energy is carried by four particles, and a lighter stop squark is involved in the decay. To separate the process from Standard Model $t \bar{t}$ production the invariant mass of each stop candidate is reconstructed. For real signal this distribution should peak lower than for heavier Standard Model $t \bar{t}$ decays. The primary challenge is in reconstructing this highly underconstrained system. The approach used is to approximate the neutrino-neutralino pairs as single pseudoparticles, and then use a $\chi^{2}$ minimization to determine the most likely momenta of each pseudoparticle. Further details can be found in the note. In the end, no excess above Standard Model predictions is observed, so limits are set as a function of four parameters: the masses of the top squark, chargino and neutralino, and the branching ratio for the chargino to neutralino decay.

\section{Top Charge Asymmetry}

At leading order, one expects the top quarks produced in the $p \bar{p} \rightarrow t \bar{t}$ process to go almost equally often in the direction of the incoming protons as the incoming antiprotons. At next to leading order, however, loop and radiation interference effects are expected to cause a roughly $5 \pm 1 \%$ asymmetry in this result. CDF has recently performed a measurement of the asymmetry with $3.2 \mathrm{fb}^{-1}$ of data in the single lepton channel [8].

Practically, it is quite challenging to reconstruct the asymmetry in the rest frame of the $t \bar{t}$ production. Instead CDF determines the asymmetry in the lab frame. The hadronically reconstructed quark is used to determine the direction of the top quark, while the sign of the lepton in the leptonically decaying top is used to determine the charge. Some corrections must be applied to the raw asymmetry measurement. First, background events passing selection have a different asymmetry than the signal. Second, the event selection used in the analysis causes a 
small bias in the asymmetry. Both of these effects must be corrected for. Finally, the reconstruction of the hadronic top mass direction leads to a smearing and a loss of directional information. This is corrected through an unfolding procedure. In the end, CDF determines an asymmetry of $A_{f b}=0.193 \pm 0.065$ (stat) \pm 0.024 (syst). This result is consistent with a $0.9 \mathrm{fb}^{-1}$ D0 result of $A_{f b}=0.12 \pm 0.08($ stat $) \pm 0.01($ syst $)[9]$.

\section{Search for $t \bar{t} H$ Production}

Due to the large mass of the top quark, the Higgs boson is expected to couple quite strongly to it. Associated production of $t \bar{t}$ with a low mass Higgs will produce a quite dramatic signature, $t \bar{t} H \rightarrow t \bar{t} b \bar{b}$. Under the Standard Model, the cross section for this process is much too small to be observed at the Tevatron, so an excess would be a clear sign of new physics.

D0 searches for this signature in the channel where the $t \bar{t}$ pair decays semi-leptonically with $2.1 \mathrm{fb}^{-1}$ of data [10]. To separate the signal and background processes they split the sample into bins based upon the number of observed jets and the number of observed $b$-tagged jets, and plot the $H_{T}$ distributions in each bin. Signal events should be expected to cluster at the high $H_{T}$ end of the distributions with a high number of jets and $b$-tags. In the end D0 observes no excess above Standard Model expectations, so they set limits on Standard Model Higgs production as well as certain more exotic physics scenarios.

\section{Searches for $t \rightarrow H^{+} b$ Decays}

In many extensions of the Standard Model, an additional Higgs doublet is required, leading to the presence of charged Higgs bosons. If the charged Higgs is light enough then it should appear in top quark decays.

The D0 Collaboration has performed direct searches for this process under supersymmetric models with $1.0 \mathrm{fb}^{-1}$ of data. For lower values of $\tan (\beta)$ the charged Higgs should decay primarily $H^{+} \rightarrow c \bar{s}$, while for higher values of $\tan (\beta)$ the primary decay mode is $H \rightarrow \tau^{+} v$. D0 has searched for both of these decay modes and combined the results [11]. Specifically, it performs counting experiments binned based upon the number of observed events in many decay channels based upon the number of observed jets, electrons, muons, taus, and $b$-tags. Since good agreement is seen with the Standard Model expectations in every channel limits are set, limiting the branching ratio of $t \rightarrow H^{+} b$ to roughly $20 \%$ at $95 \%$ confidence level for most hypothesis scenarios.

Between the D0 limits and similar limits set by the LEP collaborations, top to charged Higgs decays are unlikely to exist under most of MSSM parameter space. There are many other scenarios to be investigated, however. CDF has recently performed a search with $2.7 \mathrm{fb}^{-1}$ in the NMSSM scenario, in which the charged Higgs decays into a light, neutral Higgs boson [12]. This boson is excluded at masses above $2 m_{b}$, so CDF searches for their decays into taus: $t \rightarrow H^{+} b \rightarrow W^{+} A b \rightarrow$ $W^{+} b \tau \bar{\tau}$. Thus, when a top decays in this mode, the final state signature will be very similar to Standard Model $t \bar{t}$ production except that there will be two extra soft taus produced. In practice, CDF searches for this signature in the standard single lepton $t \bar{t}$ channel, where an extra low momentum isolated track is required. The most challenging background to understand in this search is Standard Model $t \bar{t}$ production in association with an extra track from underlying event interactions. 
As this background will be poorly modeled in simulation, CDF extracts the background from a jet-triggered data sample, and validates the shape by showing that a fit to the track $p_{T}$ spectrum returns a $Z+$ jet contribution with the proper cross section. In the end, CDF sees no excess above the Standard Model, so they set limits as a function of the hypothesis Higgs masses over the allowed kinematic ranges.

\section{Conclusion}

The experiments at the Tevatron have by now studied many aspects of the top quark signature for evidence of physics beyond the Standard Model. So far all signatures appear to be consistent with the Standard Model, however many signatures remain statistically limited. Work is ongoing to analyze additional data as it becomes available and to deepen our understanding of the top sector.

\section{References}

[1] M. Perfilov, Search for Anomalous Top Quark Production at the Tevatron, Proceedings of the XVIII Workshop on D.I.S.

[2] J. Adelman Top mass measurement at CDF, Proceedings of the XVIII Workshop on D.I.S; G. Petrillo Measurement of the top quark mass at D0, Proceedings of the XVIII Workshop on D.I.S.

[3] CDF Public Top Group Webpage: http://www-cdf.fnal.gov/physics/new/top/top.html. D0 Public Top Group Webpage: http://www-d0.fnal.gov/Run2Physics/top/top_public_web_pages/top_public.html.

[4] The D0 Collaboration, D0 Note 5882-CONF (2009).

[5] The CDF Collaboration, CDF Conference Note 9844 (2009).

[6] The D0 Collaboration, D0 Note 5937-CONF (2009).

[7] The CDF Collaboration, CDF Conference Note 9439 (2008).

[8] The CDF Collaboration, CDF Conference Note 9853 (2009).

[9] V. M. Abazov et al. (D0 Collaboration), PRL 100, 142002 (2008).

[10] The D0 Collaboration, D0 Note 5739-CONF (2009).

[11] V. M. Abazov et al. (D0 Collaboration), PLB 682, 278 (2009).

[12] The CDF Collaboration, CDF Conference Note 10104 (2010). 